\title{
ARTICLE Effect of the dopamine stabilizer (-)-OSU6162 on potentiated incubation of opioid craving after electric barrier-induced voluntary abstinence
}

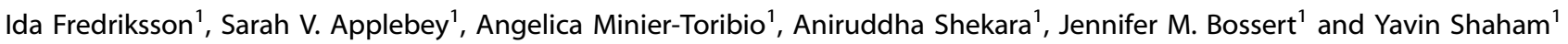

\begin{abstract}
In the classical incubation of drug craving rat model, drug seeking is assessed after homecage forced abstinence. However, human abstinence is often voluntary because negative consequences of drug seeking outweigh the desire for the drug. Here, we developed a rat model of incubation of opioid craving after electric barrier-induced voluntary abstinence and determined whether the dopamine stabilizer (-)-OSU6162 would decrease this new form of incubation. We trained male and female rats to selfadminister oxycodone $(0.1 \mathrm{mg} / \mathrm{kg} /$ infusion, $6 \mathrm{~h} / \mathrm{day})$ for 14 days. We then exposed them to either homecage forced abstinence or voluntary abstinence induced by an electric barrier of increasing intensity near the drug-paired lever. On abstinence days 1,15 , or 30 , we tested the rats for oxycodone seeking without shock and drug. We also examined the effect of (-)-OSU6162 (7.5 and $15 \mathrm{mg} /$ $\mathrm{kg}$ ) on oxycodone seeking on abstinence day 1 or after 15 days of either voluntary or forced abstinence. Independent of sex, the time-dependent increase in oxycodone seeking after cessation of opioid self-administration (incubation of opioid craving) was stronger after voluntary abstinence than after forced abstinence. In males, (-)-OSU6162 decreased incubated (day 15) but not nonincubated (day 1) oxycodone seeking after either voluntary or forced abstinence. In females, (-)-OSU6162 modestly decreased incubated oxycodone seeking after voluntary but not forced abstinence. Results suggest that voluntary abstinence induced by negative consequences of drug seeking can paradoxically potentiate opioid craving and relapse. We propose the dopamine stabilizer (-)-OSU6162 may serve as an adjunct pharmacological treatment to prevent relapse in male opioid users.
\end{abstract}

Neuropsychopharmacology (2020) 45:770-779; https://doi.org/10.1038/s41386-020-0602-6

\section{INTRODUCTION}

Since the late 1990s, prescription opioid addiction and overdose rates have dramatically increased in the US [1], underscoring the need for new treatments [2]. High relapse rates are a major contributor to the opioid crisis [1, 3, 4]. Despite this clinical problem, there are only a few preclinical publications on prescription opioid relapse [5-12]. In humans, relapse and craving are commonly triggered by reexposure to cues and contexts previously associated with drug use [13-15]. In laboratory rats, drug seeking in the presence of drug-associated cues and contexts progressively increases after cessation of drug selfadministration [16], a phenomenon termed incubation of drug craving [17]. This incubation phenomenon has been observed in rats across several drug classes $[16,18]$, including the opioid drugs heroin and oxycodone $[5-7,10,19,20]$. There is also evidence for incubation of drug craving in humans [21-24].

In the classical incubation of craving model, drug seeking is assessed after homecage forced abstinence $[16,17,25]$. However, from a translational perspective, a potential limitation of this model is that abstinence in humans is often voluntary, because the negative consequences of drug use outweigh the desire for the drug [26]. Based on this consideration, we thought to improve the homology between the human condition and the rat model by incorporating a negative consequences-induced voluntary abstinence manipulation into the classical incubation model. We used a variation of the classical electric barrier "conflict" model [27-29] that was recently adapted to study drug relapse [30-32]. In these studies, cue-induced relapse to drug seeking was determined after electric barrier-induced suppression of drug seeking [30-32].

In our modified incubation model, we induce abstinence by introducing an electric barrier of increasing intensity that causes cessation of drug self-administration. We then test for relapse to drug seeking without the barrier after different voluntary abstinence periods. The electric barrier-relapse model is conceptually similar to our recent punishment-based relapse models $[33,34]$, with one important difference: the negative consequences are associated with drug seeking prior to drug taking [30].

We first determined whether incubation of oxycodone craving would occur after electric barrier induced voluntary abstinence. We tested rats for drug seeking 1 day after cessation of oxycodone self-administration, and then after 15 or 30 days of homecage abstinence or electric barrier-induced abstinence. Next, we tested the effect of the dopamine stabilizer (-)-OSU6162 (a potential addiction treatment medication [35]) on incubated oxycodone seeking after either voluntary or forced abstinence. (-)-OSU6162 was developed by Arvid Carlsson and colleagues [36-38] and can stimulate or inhibit dopamine-related behaviors depending on dopaminergic tone. For example, (-)-OSU6162 inhibits amphetamine-induced locomotor activity but stimulates

${ }^{1}$ Behavioral Neuroscience Branch, IRP/NIDA/NIH, Baltimore, MD, USA

Correspondence: Ida Fredriksson (Ida.Fredriksson@nih.gov) or Yavin Shaham (yavin.shaham@nih.gov)

Received: 3 September 2019 Accepted: 17 December 2019

Published online: 6 January 2020 
locomotor activity in rats habituated to their environment [36-38]. In male rats, systemic (-)-OSU6162 injections decrease homecage alcohol drinking, alcohol self-administration, alcohol withdrawal symptoms, cue-induced reinstatement of alcohol seeking, and the alcohol deprivation effect $[39,40]$. Additionally, in alcohol-dependent individuals, (-)-OSU6162 decreases alcohol craving [35].

\section{MATERIALS AND METHODS}

Subjects

We used male $(n=148)$ and female $(n=142)$ Sprague-Dawley rats (Charles River), weighing 290-350 and 190-240 g prior to surgery. We maintained the rats under a reverse $12: 12 \mathrm{~h}$ light/dark cycle (8:00 a.m. lights off) with food and water freely available. We housed two rats/cage prior to surgery and then individually after surgery. We performed experiments in accordance with NIH Guide for the Care and Use of Laboratory Animals (8th edition), under a protocol approved by the local ACUC. We excluded 12 rats due to catheter failure $(n=4)$, poor health/death $(n=7)$, or failure to acquire oxycodone self-administration $(n=1)$.

\section{Drugs}

We received oxycodone hydrochloride $(\mathrm{HCl})$ from NIDA pharmacy and dissolved it in saline. We chose a unit dose of $0.1 \mathrm{mg} / \mathrm{kg}$ based on our previous study [7]. We received (-)-OSU6162 from Drs. Arvid Carlsson and Pia Steensland (Karolinska Institutet) and dissolved it in saline $(5 \mathrm{~mL} / \mathrm{kg})$. Based on previous studies, we injected (-)-OSU6162 at doses of 7.5 and $15 \mathrm{mg} / \mathrm{kg}$, s.c., $60 \mathrm{~min}$ prior to the test sessions $[36,39,40]$. We habituated the rats to saline injections for 3 days.

\section{Intravenous surgery}

We anesthetized the rats with isoflurane (5\% induction; 2-3\% maintenance, Butler Schein) and implanted silastic catheters into the jugular vein as previously described [41-43]. We injected the rats with ketoprofen ( $2.5 \mathrm{mg} / \mathrm{kg}$, s.c., Butler Schein) after surgery and the following day to relieve pain. We allowed the rats to recover for 6-8 days before training. We flushed the catheters daily with gentamicin $(4.25 \mathrm{mg} / \mathrm{mL}$; APP Pharmaceuticals) dissolved in sterile saline. If rats lost catheter patency during the training phase, we re-catheterized the other jugular vein and continued training the next day.

\section{Apparatus}

We used Med Associates chambers. Each chamber had two levers located $7.5-8 \mathrm{~cm}$ above the grid floor on opposing walls. Responding on the active retractable lever activated the infusion pump, while lever presses on the inactive, non-retractable lever had no consequences. We connected the grid floor to a shocker (Med Associates ENV-410B).

\section{Procedure}

The experiments consisted of four phases: oxycodone selfadministration training (14 days), early tests for oxycodone seeking (abstinence day 1), electric barrier-induced abstinence or homecage abstinence (13 or 28 days), and late tests for oxycodone seeking (abstinence day 15 or 30 ).

\section{Oxycodone self-administration training}

We trained the rats to self-administer oxycodone- $\mathrm{HCl}$ for $6 \mathrm{~h} /$ day (six 1 -h sessions separated by $10 \mathrm{~min}$ ) for 14 days. Oxycodone was infused at a volume of $100 \mu \mathrm{L}$ over $3.5 \mathrm{~s}$ at a dose of $0.10 \mathrm{mg} / \mathrm{kg} /$ infusion. Each session began with illumination of a red houselight that remained on for the entire session, followed $10 \mathrm{~s}$ later by the insertion of the active lever. Active lever presses led to oxycodone infusions that were paired with a 20-s tone-light cue under a fixedratio 1 (FR1) reinforcement schedule 20-s timeout. At the end of each 1-h session, the houselight turned off and the active lever retracted. We limited oxycodone intake to 15 infusions/h.

\section{Electric barrier-induced abstinence}

During this phase, oxycodone was available for $2 \mathrm{~h} /$ day. We used the same parameters (oxycodone dose, reinforcement schedule, tone-light cues, etc.) used during training. We achieved abstinence by introducing an electric barrier near the active lever ("shock zone") [30]. We separated the "shock zone" (2/3 of the chamber) from a "safe zone" (remaining 1/3 of the chamber) with a plastic demarcation (Mcmaster, cat\# 9852K61). If the rats approached the active lever, they received a continuous mild footshock (0.1-0.4 mA). On the first day, the current was set at $0.0 \mathrm{~mA}$ and was gradually increased every day by $0.1 \mathrm{~mA}$, stopping at $0.3 \mathrm{~mA}$. If rats did not suppress oxycodone self-administration, the intensity was increased to $0.4 \mathrm{~mA}$ the next day.

Homecage forced abstinence

We kept the rats in their homecage and handled them six times per week.

Relapse (incubation) tests

We tested the rats for oxycodone seeking under extinction conditions on abstinence days 1 and 15 or 30 . On day 1 , the test session was $30 \mathrm{~min}$ to minimize extinction learning/experience, which may decrease incubated drug seeking during testing on day 15 or 30 . During testing, we turned off the electric barrier and removed the plastic demarcation. We gave all rats a $30 \mathrm{~min}$ habituation period in the self-administration chamber before the start of the test session to allow them to realize that the barrier is not electrified. Lever presses resulted in delivery of the oxycodone-paired tone-light cue and activation of the infusion pump, but no drug infusions.

Exp 1: Effect of electric barrier-induced voluntary abstinence on incubation of oxycodone craving. We compared incubation of oxycodone craving after homecage forced abstinence (the classical incubation model $[16,17,25]$ ) with incubation of oxycodone craving after electric barrier-induced abstinence (voluntary abstinence). We used eight groups of rats ( $n=12-14$ per group) in a mixed experimental design that included the between-subjects factors of Sex and Abstinence Condition (forced, electric barrier), and the within-subjects factor of Abstinence Day (1, 15 or 1, 30) (Fig. 1a). We matched the different groups for total oxycodone infusions during the training phase. Prior to the electric barrier phase, we tested the rats' sensitivity to footshock (operationally defined as the minimal shock level that causes the withdrawal of the front paw): males: 0.16 $\mathrm{mA} \pm 0.005$; females: $0.18 \mathrm{~mA} \pm 0.005, p=0.047$ ).

Exp 2: Effect of (-)-OSU6162 on incubation of oxycodone craving after electric barrier-induced abstinence. We determined the effect of systemic injections of vehicle (saline) or (-)-OSU6162 (7.5 or 15 $\mathrm{mg} / \mathrm{kg}$, s.c) on incubation of oxycodone craving after electric barrier-induced abstinence. We used 12 groups of rats $(n=9-15$ per group) in an experimental design that included the betweensubjects factors of Abstinence Day $(1,15)$, Sex, and (-)-OSU6162 Dose $(0,7.5,15 \mathrm{mg} / \mathrm{kg})$, and the within-subjects factor of Session Time (30, 60, 90 min) (Fig. 2a). We matched the different groups for total oxycodone infusions during the training phase. To verify that incubation had occurred, we injected all rats with saline and tested them for drug seeking on day 1 in a 30-min session. We compared the number of lever presses on the day 1 test to the number of lever presses during the first $30 \mathrm{~min}$ of the day 15 test in rats injected with saline on both days. These rats showed robust incubation of oxycodone craving: $41.9 \pm 5.6$ and $87.6 \pm 5.7$ lever presses/30 $\mathrm{min}$ for day 1 and day 15 , respectively, $n=27, p<0.05$. Unlike Exp. 1, there were no sex differences in shock sensitivity: males: $0.16 \mathrm{~mA} \pm 0.003$; females: $0.17 \mathrm{~mA} \pm 0.009, p>0.1$ ). 
Exp 3: Effect of (-)-OSU6162 on incubation of oxycodone craving after forced abstinence. We tested the specificity of the effect of (-)-OSU6162 on incubated oxycodone seeking after electric barrier-induced abstinence by determining the effect of vehicle (saline) or (-)-OSU6162 (15 mg/kg, S.c.) on incubation of oxycodone craving after forced abstinence. We used four groups of rats ( $n=9-11$ per group) in an experimental design that included the between-subjects factors of (-)-OSU6162 Dose $(0,15 \mathrm{mg} / \mathrm{kg})$ and Sex, and the within-subjects factor of Session Time (30, 60, $90 \mathrm{~min})$ (Fig. 3a). We matched the different groups for total oxycodone infusions during the training phase. The experimental phases were the same as in Exp. 2, except that we exposed the rats to forced abstinence in the homecage before the oxycodone-seeking test on abstinence day 15 . To verify that incubation had occurred, we injected all rats with saline and tested them for drug seeking on day 1 in a 30-min session. We compared the number of lever presses on the day 1 test to the number of lever presses during the first $30 \mathrm{~min}$ of the day 15 test in rats injected with saline on both days. These rats showed incubation of oxycodone craving: $33.5 \pm 4.12$ and $72.8 \pm 6.2$ lever presses/30 $\min$ for day 1 and day $15, n=20, p<0.01$.

Exp 4: Effect of (-)-OSU6162 on oxycodone self-administration. At the end of Exp. 2, we tested the specificity of the effect of (-)-OSU6162 on incubated oxycodone seeking in rats by determining the drug's effect on ongoing oxycodone selfadministration $(0.1 \mathrm{mg} / \mathrm{kg} /$ infusion) in male $(n=6)$ and female $(n=7)$ rats. We used two groups of rats in an experimental design that included the between-subjects factor of Sex and the withinsubjects factor of (-)-OSU6162 Dose $(0,7.5,15 \mathrm{mg} / \mathrm{kg}$ ) (see experimental timeline Fig. 4a). We first retrained the rats on the FR1-20-s timeout reinforcement schedule for 3 days and then tested them (counterbalanced within-subjects design) after pretreatment ( $60 \mathrm{~min}$ ) with saline, $7.5 \mathrm{mg} / \mathrm{kg}$, and $15 \mathrm{mg} / \mathrm{kg}$ over 3 days. We then repeated this cycle using a progressive ratio reinforcement schedule [44]. For each rat, the program turned off $60 \mathrm{~min}$ after the last oxycodone infusion.

Statistical analyses

We analyzed the data with ANOVAs or ANCOVAs (inactive lever as a covariate) using SPSS (Version 23, GLM procedure). We followed significant main effects and interactions $(p<0.05)$ with post hoc tests (Fisher's PLSD). We describe the different between- and within-subjects factors for the different analyses in the Results section. Because the multifactorial ANCOVAs yielded multiple main and interaction effects, we only report significant effects that are critical for data interpretation. For clarity, we indicate the results of post hoc analyses with asterisks in the figures, but do not describe them in the Results section. For a complete reporting of the statistical analyses see Table S1, and for individual data of the bar graphs described in Figs. 1-4 see Fig. S1. The $n$ per group in the different experiments is based on our previous incubation of craving studies [41, 42, 45, 46].

\section{RESULTS}

Oxycodone self-administration (Exp. 1-3)

The timeline of Exp. 1-3 is provided in Figs. 1a, 2a, and 3a. The male and female rats demonstrated reliable oxycodone selfadministration (Figs. 1b, 2b, and 3b). There were no sex differences in oxycodone self-administration in Exp. 1 or Exp. 3. In Exp. 2, oxycodone self-administration was higher in males than in females (Sex $\left[F_{1,132}=4.5, p=0.035\right.$; Sex by Session $\left[F_{13,1716}=\right.$ 2.4, $p=0.003])$. The complete analyses for number of infusions and active and inactive lever presses during training are described in Table S1.
Electric barrier-induced voluntary abstinence (Exp. 1 and 2) Male and female rats voluntarily abstained from drug selfadministration when we introduced an electric barrier of increasing shock intensity near the active lever (Figs. 1c and 2c). There were no sex differences in electric barrier-induced voluntary abstinence. The mean number of infusions for the last 3 days of electric barrier-induced abstinence was below 1 per session. In Exp. 1 and 2, 28 and 25 of the 105 and 134 rats required exposure to $0.4 \mathrm{~mA}$ to eliminate oxycodone self-administration. The statistical analyses during electric barrier-induced abstinence are described in Table S1.

Effect of electric barrier-induced voluntary abstinence on incubation of oxycodone craving. The goal of Exp. 1 was to determine whether incubation of oxycodone craving would occur after electric barrier-induced voluntary abstinence. For this purpose, we tested the rats after different periods of homecage forced abstinence or electric barrier-induced voluntary abstinence (15 or 30 days). We tested all rats for oxycodone seeking under extinction conditions 1 day after oxycodone self-administration training and then tested them again on either day 15 or day 30 .

Oxycodone seeking in the relapse (incubation) tests was greater after 15 or 30 abstinence days than after 1 day, demonstrating incubation of oxycodone craving after both forced and electric barrier-induced abstinence. More importantly, this incubation effect was stronger after electric barrier-induced abstinence than after forced abstinence (Fig. 1d). The ANCOVA for number of active lever presses of rats tested on days 1 and 15 or days 1 and 30, which included the between-subjects factors of Sex and Abstinence Condition (forced, electric barrier) and the within-subjects factor of Abstinence Day (1, 15 or 1,30), showed significant Abstinence Day $\times$ Abstinence Condition interactions: $F_{1,48}=19.2, p$ $<0.001$ for the comparison of days 1 and 15 , and $F_{1,47}=11.0, p<$ 0.002 for the comparison of days 1 and 30. There were no significant effects of Sex or interactions with Sex (see Table S1).

The results of Exp. 1 demonstrate a sex-independent potentiation of incubation of oxycodone craving after electric barrierinduced voluntary abstinence.

Effect of (-)-OSU6162 on incubation of oxycodone craving after electric barrier-induced abstinence. The goal of Exp. 2 was to determine whether the dopamine stabilizer (-)-OSU6162, which decreases alcohol relapse in rat models [39, 40] and alcohol craving in humans [35], would decrease incubation of oxycodone craving after electric barrier-induced abstinence. For this purpose, we tested different groups of male and female rats for the effect of saline or (-)-OSU6162 on oxycodone seeking 1 day after oxycodone self-administration or after 15 days of electric barrierinduced abstinence.

Pretreatment with (-)-OSU6162 decreased incubated oxycodone seeking on day 15 but had no effect on non-incubated oxycodone seeking on day 1 (Fig. 2c). The effect on day 15 was somewhat stronger for males and selective for the first $30 \mathrm{~min}$ of testing. The ANCOVA for number of active lever presses included the between-subjects factors of Abstinence Day, Sex, and OSU6162 Dose $(0,7.5,15)$, and the within-subjects factor of Session Time $(30,60,90)$, showed significant effects of Abstinence Day $\times$ OSU6162 Dose $\left(F_{2,121}=4.1, p=0.019\right)$ and Abstinence Day $\times$ OSU6162 Dose $\times$ Session Time $\left(F_{4,242}=9.8, \quad p<0.001\right)$. There was no significant effect of Sex or interactions with Sex. We also performed separate analyses for males and females, because the effect of (-)-OSU6162 appeared stronger and dosedependent in males (Fig. 2c). For males, the ANCOVA of active lever presses showed significant effects of Abstinence Day $\times$ OSU6162 Dose $\left(F_{2,61}=4.1, p=0.022\right)$ and Abstinence Day $\times$ OSU6162 Dose $\times$ Session Time $\left(F_{4,122}=6.9, \quad p<0.001\right)$. For females, only the triple interaction was significant $\left(F_{4,22}=\right.$ $3.4, p=0.011$ ). 


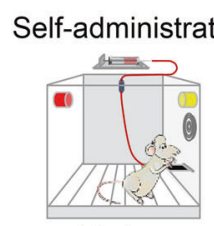

14 days

\section{A. Experimental timeline}

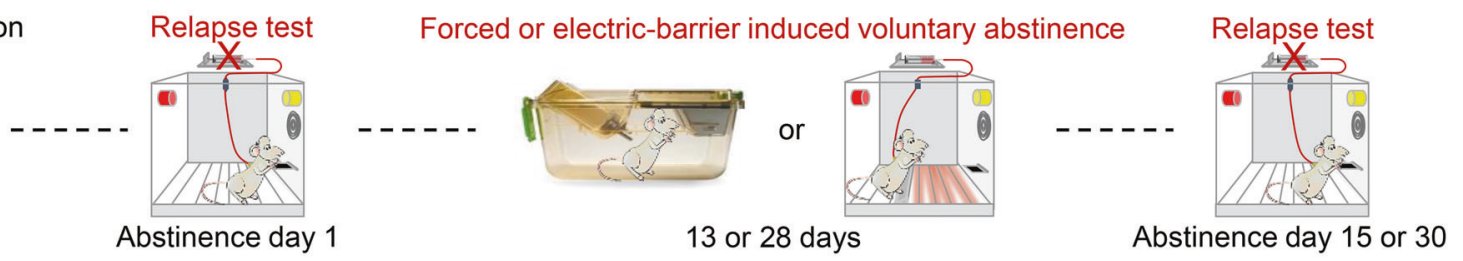

B. Self-administration
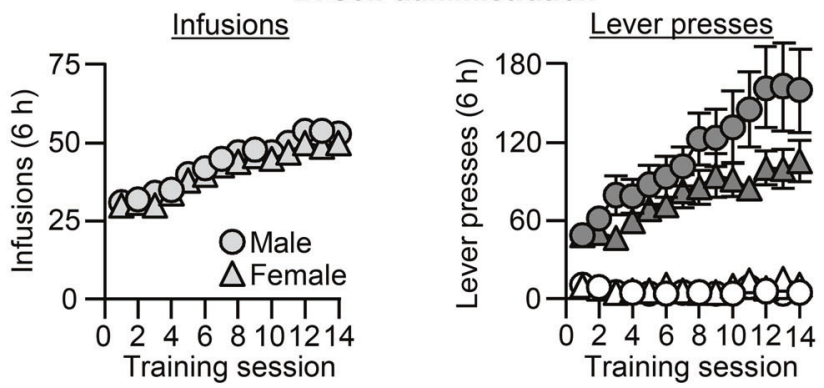

O Male: inactive lever

Male: active lever

$\triangle$ Female: inactive lever

$\triangle$ Female: active lever

\section{Electric barrier-induced abstinence}
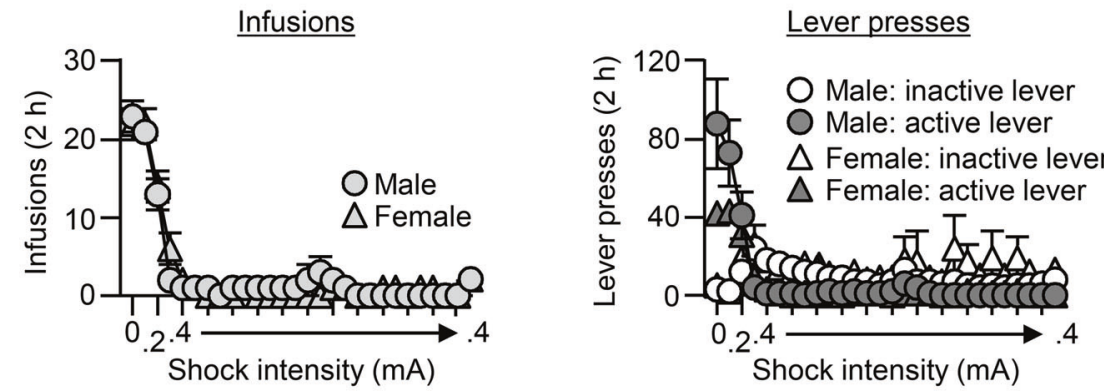

D. Relapse (incubation) tests



Fig. 1 Effect of electric barrier-induced voluntary abstinence on incubation of oxycodone craving. a Timeline of Exp. 1. b Oxycodone selfadministration training: Number of oxycodone infusions $(0.1 \mathrm{mg} / \mathrm{kg} / \mathrm{infusion})$ and inactive and active lever presses during the $6-\mathrm{h}$ sessions. c Electric barrier-induced abstinence: Number of oxycodone infusions and inactive and active lever presses during the 2-h sessions. $\mathbf{d}$ Relapse (incubation) tests: Number of active lever presses during the 30-min test sessions. During testing, active lever presses led to contingent presentation of the tone-light cue previously paired with oxycodone infusions during training, but not oxycodone infusions (extinction conditions). We tested separate groups of rats on either days 1 and 15, or days 1 and 30 (within-subjects testing). There were no group differences on day 1 testing in the two experimental conditions. Thus, for clarity of data presentation we combined the day 1 data of the two within-subjects test conditions. ${ }^{*}$ Different from day $1 .{ }^{\#}$ Different from forced abstinence group on day 15 or day $30, p<0.05$. Data are mean \pm SEM. Forced abstinence: day $1, n=28$ males/25 females; day 15, 14 males/13 females; and day 30, 14 males/12 females. Electric barrierinduced abstinence: day 1, 28 males/24 females; day 15, 14 males/12 females; and day 30, 14 males/12 females. See Fig. S1 for individual data.

The results of Exp. 2 demonstrate that (-)-OSU6162 selectively decreased incubated oxycodone seeking after electric barrierinduced abstinence. This effect was selective for the first $30 \mathrm{~min}$ of the test session and somewhat stronger in males.

Effect of (-)-OSU6162 on incubation of oxycodone craving after forced abstinence. The goal of Exp. 3 was to determine whether the effect of (-)-OSU6162 on incubated oxycodone seeking after voluntary abstinence due to negative consequences would generalize to incubation after homecage forced abstinence. We tested different groups of male and female rats for the effect of saline or (-)-OSU6162 $(15 \mathrm{mg} / \mathrm{kg})$ on oxycodone seeking after 15 days of forced abstinence.

Pretreatment with (-)-OSU6162 decreased incubated oxycodone seeking on day 15 in males but not in females (Fig. 3). The mixed factorial ANCOVA for number of active lever presses, which 
A. Experimental timeline

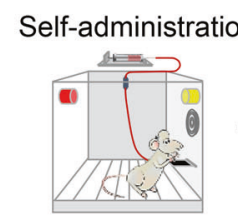

14 days

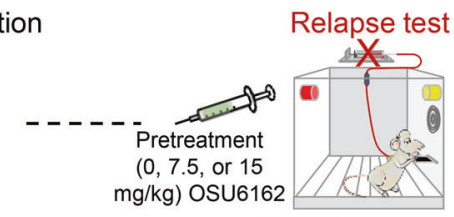

Abstinence day 1

Electric-barrier induced abstinence

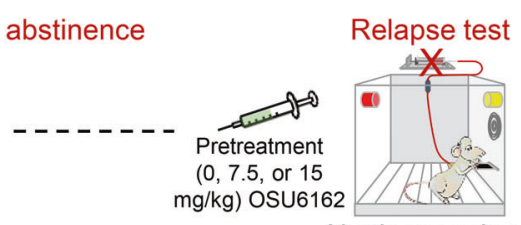

Abstinence day 15

\section{B. Self-administration}

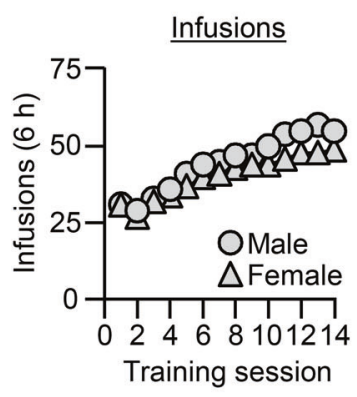

Lever presses



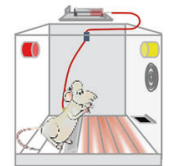

13 days $\mathrm{mg} / \mathrm{kg}$ ) OSU61

\section{nce} Infusions

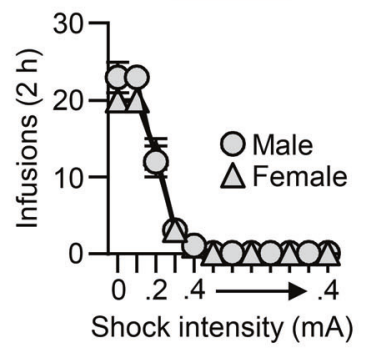

Lever presses

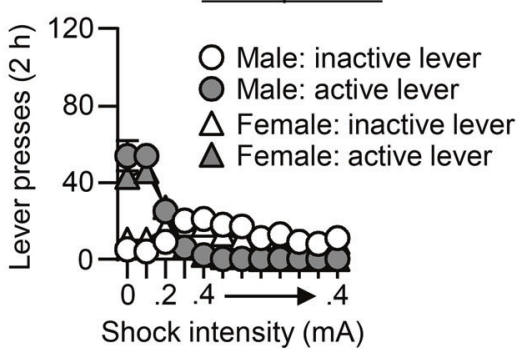

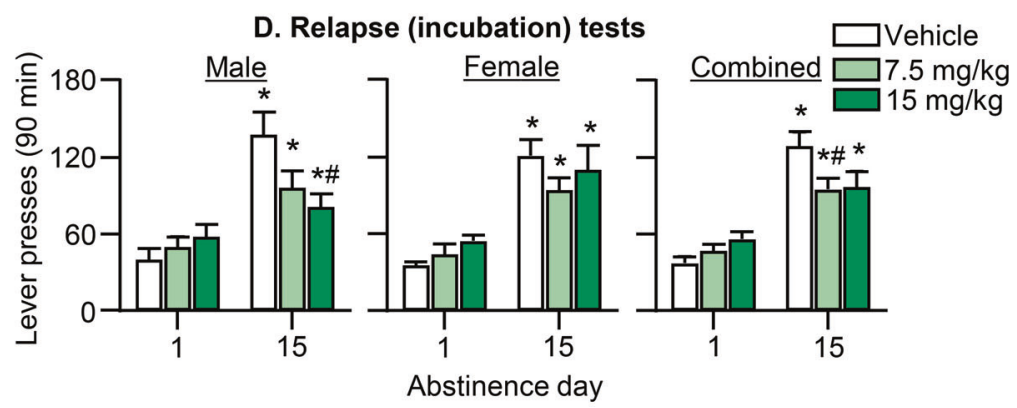

E. Relapse (incubation) day 1 time course



F. Relapse (incubation) day 15 time course

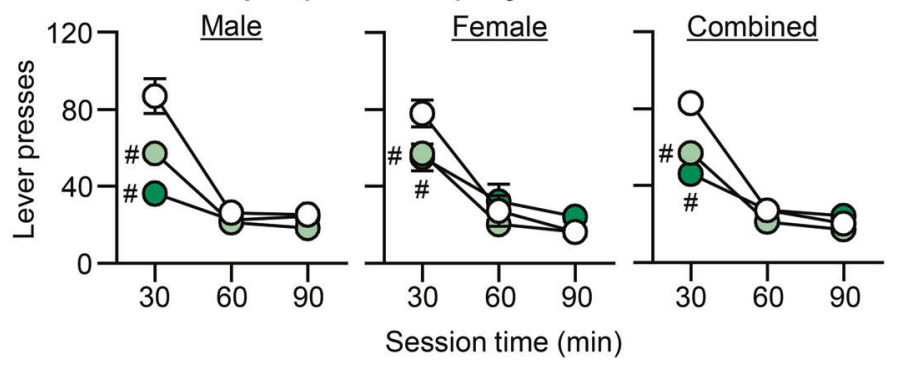

Fig. 2 Effect of (-)-OSU6162 on incubation of oxycodone craving after electric barrier-induced abstinence. a Timeline of Exp. 2. b Oxycodone self-administration training: Number of oxycodone infusions and inactive and active lever presses during the 6-h sessions. c Electric barrier-induced abstinence: Number of oxycodone infusions and inactive and active lever presses during the 2-h sessions. $\mathbf{d}$ Relapse (incubation) tests: Total responding. Number of active lever presses during the 90-min test sessions after (-)-OSU6162 pretreatment $(0,7.5,15 \mathrm{mg} / \mathrm{kg})$. e, $\mathbf{f}$ Relapse (incubation) tests: Time course day 1 and 15. 30-min time course of active lever presses 1 day after oxycodone self-administration training and after 13 days of electric barrier-induced voluntary abstinence. * Different from day 1 within each dose condition; " Different from the vehicle group on day 15, $p<0.05$. Data are mean \pm SEM; Day 1, 9-10 males/dose and 9 females/dose; Day 15, 10-13 males/dose and 10-15 females/dose. See Fig. S1 for individual data. 


\section{A. Experimental timeline}
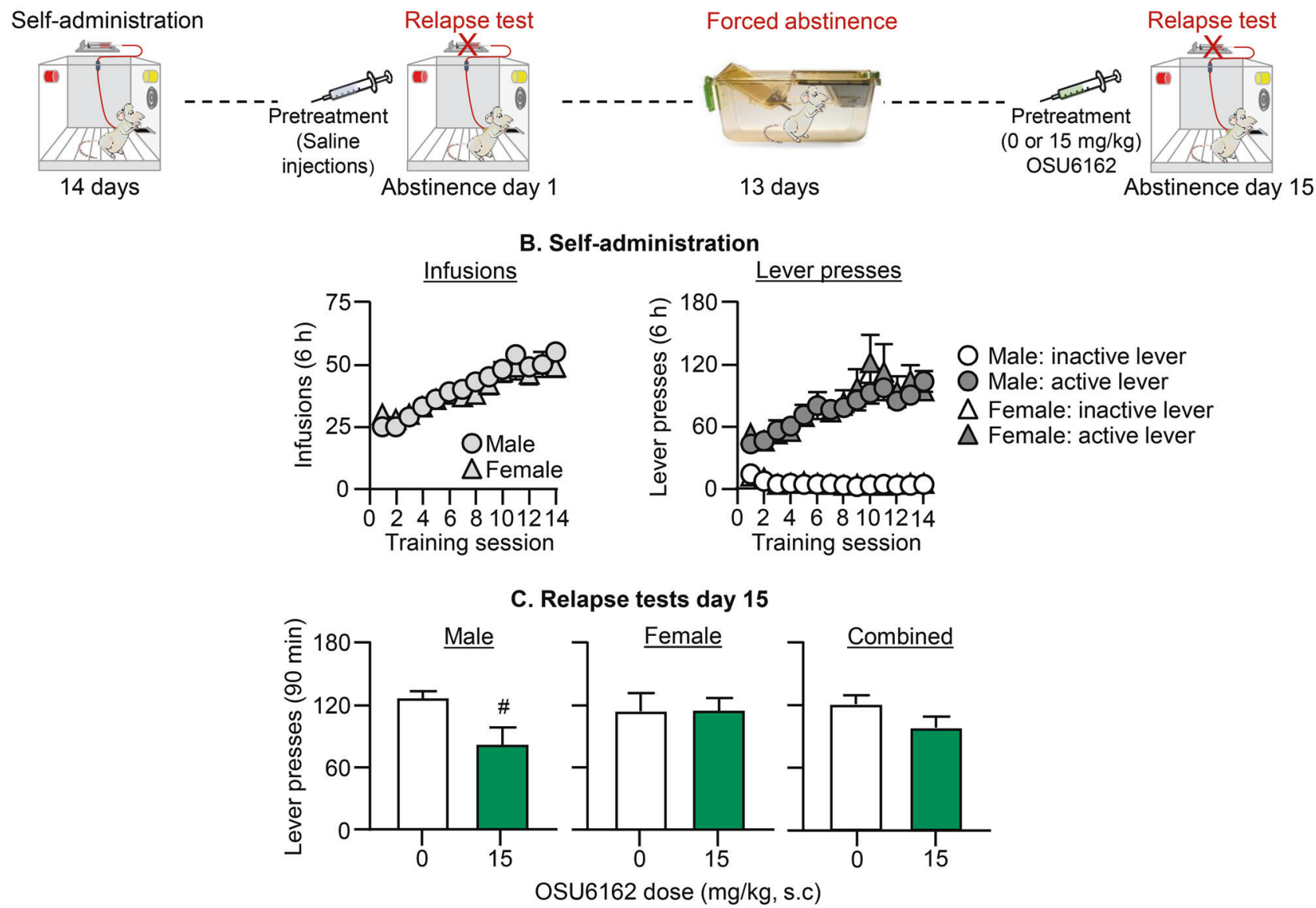

D. Relapse tests day 15 time course

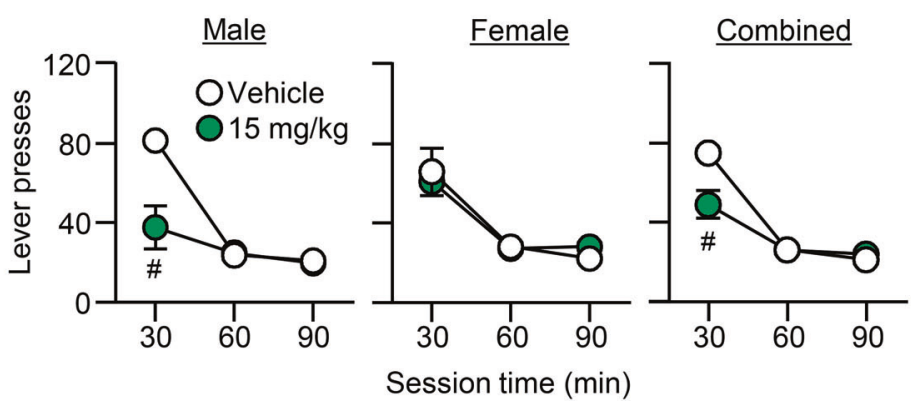

Fig. 3 Effect of (-)-OSU6162 on incubation of oxycodone craving after forced abstinence. a Timeline of Exp. 3. b Oxycodone selfadministration training: Number of oxycodone infusions and inactive and active lever presses during the 6-h sessions. c Relapse tests: Total responding. Number of active lever presses during the 90 -min test sessions after (-)-OSU6162 pretreatment $(0,15 \mathrm{mg} / \mathrm{kg})$. d Relapse tests: Time course. 30-min time course of active lever presses after 13 days of forced abstinence. " Different from the vehicle group, $p<0.05$. Data are mean \pm SEM; 11-10 males/dose and 9 females/dose. See Fig. S1 for individual data.

included the between-subjects factors of Sex and OSU6162 Dose $(0,15)$, and the within-subjects factor of Session Time, showed significant effects of OSU6162 Dose $\times$ Session Time $\left(F_{2,68}=9.0, p\right.$ $<0.001)$ and OSU6162 Dose $\times$ Sex $\times$ Session Time $\left(F_{2,68}=4.6, p=\right.$ $0.014)$. Subsequent analysis within each sex showed significant effects of OSU6162 Dose $\left(F_{1,18}=5.7, p=0.028\right)$ and OSU6162 Dose $\times$ Session Time $\left(F_{2,36}=15.4, p<0.001\right)$ for males, but not for females ( $p$ values $>0.1$ ).

The results of Exp. 3 demonstrate that (-)-OSU6162 decreased incubated oxycodone seeking after forced abstinence in males but not in females. This effect was selective for the first $30 \mathrm{~min}$ of the test session.
Effect of (-)-OSU6162 on ongoing oxycodone self-administration. In Exp. 4, we tested a subset of male and female rats from Exp. 2 for the effect of (-)-OSU6162 on oxycodone self-administration under the FR1-20-s timeout and PR reinforcement schedules. We found that (-)-OSU6162 had no effect on either measure (see Fig. 4 and Table S1 for statistical results).

\section{DISCUSSION}

Our goal was to develop an incubation of opioid craving model that would mimic the human condition of voluntary abstinence that occurs when the negative consequences of drug seeking 


\section{A. Experimental timeline}

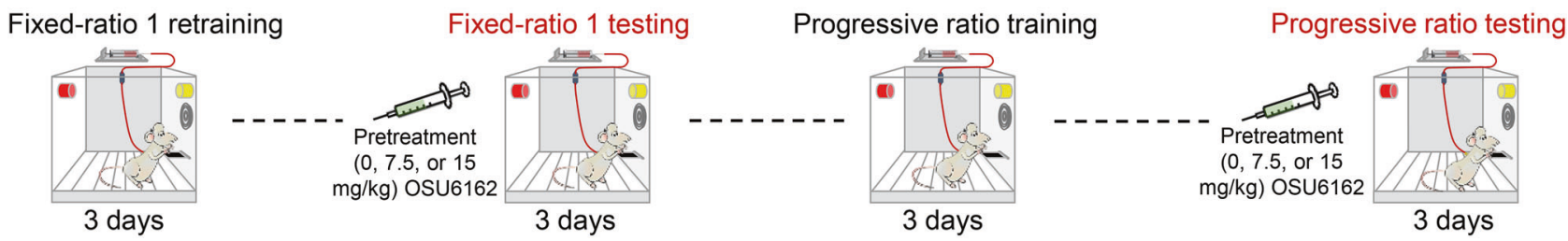

B. Self-administration
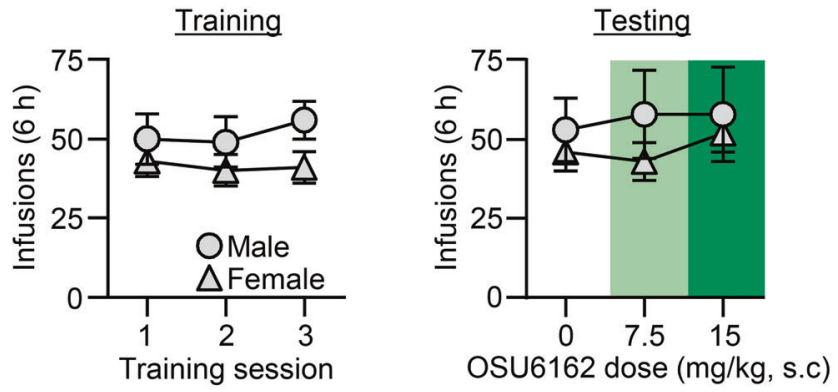

\section{Progressive ratio}
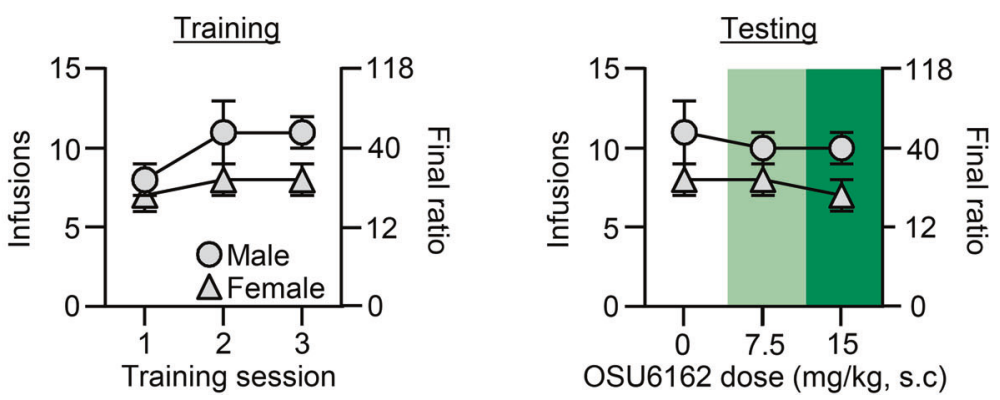

Fig. 4 Effect of (-)-OSU6162 on ongoing oxycodone self-administration. a Timeline of Exp. 4. b FR1 oxycodone self-administration training and testing (6-h sessions): Number of oxycodone infusions ( $0.1 \mathrm{mg} / \mathrm{kg} /$ infusion) after (-)-OSU6162 pretreatment $(0,7.5,15 \mathrm{mg} / \mathrm{kg})$. c PR oxycodone self-administration training and testing: Number of oxycodone infusions and final ratio after (-)-OSU6162 pretreatment (0, 7.5, $15 \mathrm{mg} / \mathrm{kg}$ ). Data are mean \pm SEM, $n=6$ males $/ 7$ females that previously participated in Exp. 2. See Fig. S1 for individual data.

outweigh the desire for the drug. Our main finding is that in both sexes, incubation of oxycodone craving was stronger after electric barrier-induced voluntary abstinence than after forced abstinence in the homecage. In an initial pharmacological characterization, we found that the dopamine stabilizer (-)-OSU6162 selectively decreased incubated oxycodone seeking after electric-barrierinduced voluntary abstinence, an effect that was somewhat stronger in males than in females. (-)-OSU6162 also decreased incubated oxycodone seeking after forced abstinence in males but not in females. (-)-OSU6162 had no effect on non-incubated drug seeking during early abstinence or on oxycodone self-administration, indicating that (-)-OSU6162's effect on incubated oxycodone seeking is not due to non-specific disruption of operant responding. Finally, in agreement with our previous heroin studies $[45,47,48]$, we found no evidence for sex differences in oxycodone self-administration. These data do not support the accepted notion that females are more vulnerable to drug selfadministration than males [49].

The electric barrier-induced voluntary abstinence relapse/ incubation model and the human condition

In humans, abstinence often occurs not because drugs are unavailable, but because they become associated with negative consequences that outweigh their rewarding effects [26]. In the classical rat incubation of craving model (and other relapse/ reinstatement models $[25,50])$ there are no negative consequences for drug-taking behaviors $[16,17,25]$. This lack of homology between the animal models and the human condition may limit their ability to identify new treatments or their predictive validity [51-53].

In an attempt to more closely mimic the human condition, we and others have developed punishment-based [54, 55] relapse models where drug taking is suppressed by a contingent footshock delivered immediately after lever pressing and drug delivery $[33,34,56-60]$. However, the punishment-based relapse models also diverge from the human condition, because in humans the negative consequences associated with drug-taking behavior rarely coincide with acute drug intoxication [61]. Instead, the negative consequences are typically either delayed (which cannot be modeled in rats because they are unable to learn punishment contingencies that are delayed by more than a few minutes [62]) or occurs during drug seeking (e.g., secure money to obtain illegal drug, stressful interactions with a drug dealer, considering delayed negative consequences) and before drug taking [30,61].

To mimic the negative components of human drug seeking, Cooper et al. [30] developed the electric-barrier conflict model of drug relapse. Their model was inspired by the classical "conflict" model that was used many years ago to assess rats' motivation to obtain non-drug rewards, including food, sex, water, and brain stimulation [27-29].

Here, we used the electric barrier to achieve voluntary abstinence within the context of the rat incubation of drug craving model [16-18]. Unexpectedly, we found that this manipulation increased incubation of opioid craving one day 
after the removal of the electric barrier. The reasons for this potentiated effect are unknown but our results agree with previous studies showing that stress exposure increases drug seeking $[63,64]$, including a recent demonstration that repeated restraint stress exposure during forced abstinence increases incubation of cocaine craving [65]. However, our findings contrast with an earlier study showing that 2 days after 9-10 days of punishment-induced abstinence, lever presses in the relapse test were very low, and incubation of methamphetamine craving emerged after 3 weeks of homecage forced abstinence [34].

What might account for immediate potentiation of drug seeking after electric barrier-induced abstinence versus inhibition of drug seeking immediately after punishment-induced abstinence? We propose a critical reason is the difference in the operant contingency of shock exposure. In the punishment but not the electric barrier procedure, shock exposure is contingent on lever pressing. This shock contingency can cause devaluation of not only the rewarding effects of the self-administered drug, but also devaluation of the appetitive motivational states induced by exposure to the discrete drug-associated cues and the lever itself [34], which likely control drug seeking during the drug-free incubation (relapse) test. This putative devaluation process would cause low drug seeking immediately after punishment-imposed abstinence. In contrast, this devaluation process is unlikely to occur after electric barrier-induced abstinence, because neither lever presses nor the discrete cues are paired with shock delivery. Consequently, the psychological processes underlying incubation of craving will remain intact (or potentiated) after electric barrier-imposed abstinence, resulting in high lever presses during the late abstinence relapse tests.

Krasnova et al. [34] proposed that the low responding 2 days after punishment-based abstinence is because prior pairings of shock with the self-administration chamber may have made the chamber a conditioned inhibitor of operant responding during the relapse tests. However, this explanation seems unlikely, because such learning process would also cause low responding immediately after cessation of electric barrier-induced abstinence.

Another reason for the different effects of electric barrier and punishment-induced abstinence on lever presses the day after termination of the aversive stimulus might be the different drugs used in our study and the Krasnova study (methamphetamine). In this regard, there is evidence that different psychological mechanisms contribute to opioid versus psychostimulant reward and relapse [66-68].

Effect of (-)-OSU6162 on incubation of oxycodone craving In our initial pharmacological characterization of incubation of oxycodone craving after electric barrier-induced abstinence, we found that (-)-OSU6162 decreased this form of incubation, an effect that was stronger in male rats. (-)-OSU6162 also decreased incubation of craving after forced abstinence in males but not females. (-)-OSU6162's effect on oxycodone seeking was only observed on abstinence day 15 but not day 1 , suggesting a selective effect on incubated opioid seeking that at least in males is independent on the method used to achieve abstinence. Our data extend recent findings showing that (-)-OSU6162 decreases cue-induced reinstatement of alcohol seeking and the alcohol deprivation effect in male rats $[39,40]$.

(-)-OSU6162 can stabilize dopamine activity depending on the dopaminergic tone [36-38], but the cellular mechanisms involved are unknown [69]. Also unknown are the brain sites mediating the effect of (-)-OSU6162 on alcohol taking and seeking in previous studies, and on incubation of oxycodone craving in the present study. Previous studies indicate that both the mesolimbic and nigrostriatal dopamine systems are involved in cue- and contextinduced reinstatement of heroin seeking after extinction or relapse after forced abstinence [70, 71]. Thus, we speculate that these systems are likely involved in the systemic effect of (-)-OSU6162 on incubation of oxycodone craving in male rats.
The positive results with (-)-OSU6162 in male rats agree with previous studies showing a role of dopamine in relapse/ reinstatement of heroin seeking in male rats [10, 72]. Pharmacological studies showed that different dopamine antagonists decrease reinstatement of opioid seeking induced by drug priming $[12,73,74]$, discrete [75-77] and contextual cues [78], and stress [74]. However, it has been shown that different opioid agonists differ in their effect on dopaminergic transmission. For example, oxycodone but not morphine or heroin robustly increases striatal dopamine levels $[79,80]$. Thus, a question for future research is whether the effect of (-)-OSU6162 on incubated oxycodone seeking extends to heroin and other opioids. Another question for future research is whether (-)-OSU6162 would also decrease incubation of oxycodone craving at longer abstinence periods (e.g. day 30 or longer).

An unresolved issue in our study is the reason for the apparent sex-specific effect of (-)-OSU6162 on incubated oxycodone seeking. To our knowledge, studies on the effect of dopaminergic drugs on reinstatement/relapse in female rats are not yet published [10]. The lack of effect in female rats may be due to sex differences in dopamine function and responses to dopaminergic drugs [81-83], or potential sex differences in (-)-OSU6162 pharmacokinetics. Finally, the negative results for the effect of (-)-OSU6162 on oxycodone self-administration under the FR1 and progressive ratio reinforcement schedules agree with negative results from previous pharmacological and lesion studies showing a minimal role of dopamine in heroin self-administration [67, 84-88].

\section{CONCLUSIONS}

We introduce a rat model that can be used to study mechanisms of incubation of opioid craving under conditions that mimic the human condition of voluntary abstinence due to negative consequences of drug seeking. The main finding in our study is the potentiation of incubation of opioid craving after electric barrier-induced voluntary abstinence. These findings contrast with our recent studies on inhibition of incubation of opioid craving after voluntary abstinence induced by offering the rats a mutually exclusive choice between the opioid drug versus palatable food [45] or social interaction [48]. A potential implication of our contrasting findings with the electric barrier versus the choiceinduced voluntary abstinence models is that abstinence induced by negative consequences in humans would paradoxically increase relapse vulnerability while abstinence induced by alternative non-drug rewards would have an opposite effect.

Finally, Khemiri et al. [35] reported that (-)-OSU6162 decreases alcohol craving in alcohol-dependent individuals, and human studies for other indications showed that the drug is safe and lacks the extrapyramidal side-effects of classical D2 antagonists $[69,89-92]$. We propose that (-)-OSU6162 may serve as an adjunct pharmacological treatment, in combination with methadone or buprenorphine maintenance therapy, to decrease relapse after prolonged abstinence in male opioid users.

\section{FUNDING AND DISCLOSURE}

The research was supported by the Intramural Research Program of NIDA (PI: Y.S.). The authors declare no competing interests.

Reporting summary

Further information on research design is available in the Nature Research Reporting Summary linked to this article.

\section{ACKNOWLEDGEMENTS}

We thank Hannah Korah, Trinity Russell, Olivia Lofaro, and Jennifer Hoots for help with surgeries. We also thank Professors Arvid Carlsson and Pia Steensland 
(Karolinska Institutet) for providing (-)-OSU6162. The paper is dedicated to Professor Arvid Carlsson who passed away on 29 June 2018.

\section{ADDITIONAL INFORMATION}

Supplementary Information accompanies this paper at (https://doi.org/10.1038/ s41386-020-0602-6).

Publisher's note Springer Nature remains neutral with regard to jurisdictional claims in published maps and institutional affiliations.

\section{REFERENCES}

1. Rudd RA, Aleshire N, Zibbell JE, Gladden RM. Increases in drug and opioid overdose deaths-United States, 2000-2014. MMWR Morb Mortal Wkly Rep. 2016;64:1378-82.

2. Epstein DH, Heilig $M$, Shaham Y. Science-based actions can help address the opioid crisis. Trends Pharmacol Sci. 2018;39:911-16.

3. Nunes EV, Gordon M, Friedmann PD, Fishman MJ, Lee JD, Chen DT, et al. Relapse to opioid use disorder after inpatient treatment: Protective effect of injection naltrexone. J Subst Abuse Treat. 2018;85:49-55.

4. Volkow ND, Collins FS. The role of science in addressing the opioid crisis. N Engl J Med. 2017;377:391-94.

5. Blackwood CA, Hoerle R, Leary M, Schroeder J, Job MO, McCoy MT, et al. Molecular adaptations in the rat dorsal striatum and hippocampus following abstinence-induced incubation of drug seeking after escalated oxycodone selfadministration. Mol Neurobiol. 2019;56:3603-15.

6. Blackwood CA, Leary M, Salisbury A, McCoy MT, Cadet JL. Escalated oxycodone self-administration causes differential striatal mRNA expression of FGFs and IEGs following abstinence-associated incubation of oxycodone craving. Neuroscience. 2019;415:173-83.

7. Bossert JM, Hoots JK, Fredriksson I, Adhikary $S$, Zhang $M$, Venniro $M$, et al. Role of mu, but not delta or kappa, opioid receptors in context-induced reinstatement of oxycodone seeking. The European journal of neuroscience. 2019;50:2075-85.

8. Leri F, Burns LH. Ultra-low-dose naltrexone reduces the rewarding potency of oxycodone and relapse vulnerability in rats. Pharmacol Biochem Behav. 2005;82:252-62.

9. Neelakantan H, Holliday ED, Fox RG, Stutz SJ, Comer SD, Haney M, et al. Lorcaserin suppresses oxycodone self-administration and relapse vulnerability in rats. ACS Chem Neurosci. 2017:8:1065-73.

10. Reiner DJ, Fredriksson I, Lofaro OM, Bossert JM, Shaham Y. Relapse to opioid seeking in rat models: behavior, pharmacology and circuits. Neuropsychopharmacology. 2019;44:465-77.

11. You ZB, Bi GH, Galaj E, Kumar V, Cao J, Gadiano A, et al. Dopamine D3R antagonist VK4-116 attenuates oxycodone self-administration and reinstatement without compromising its antinociceptive effects. Neuropsychopharmacology. 2019;44:1415-24.

12. You ZB, Gao JT, Bi GH, He Y, Boateng C, Cao J, et al. The novel dopamine D3 receptor antagonists/partial agonists CAB2-015 and BAK4-54 inhibit oxycodone-taking and oxycodone-seeking behavior in rats. Neuropharmacology. 2017;126:190-99.

13. O'Brien CP, Childress AR, McLellan AT, Ehrman R. Classical conditioning in drug dependent humans. Ann N Y Acad Sci. 1992;654:400-15.

14. Wikler A. Dynamics of drug dependence. Implications of a conditioning theory for research and treatment. Arch Gen Psychiatry. 1973;28:611-6.

15. Sinha R. New findings on biological factors predicting addiction relapse vulnerability. Curr Psychiatry Rep. 2011;13:398-405.

16. Pickens CL, Airavaara M, Theberge F, Fanous S, Hope BT, Shaham Y. Neurobiology of the incubation of drug craving. Trends Neurosci. 2011;34:411-20.

17. Grimm J, Hope B, Wise R, Shaham Y. Neuroadaptation - Incubation of cocaine craving after withdrawal. Nature. 2001;412:141-42.

18. Wolf ME. Synaptic mechanisms underlying persistent cocaine craving. Nat Rev Neurosci. 2016;17:351-65.

19. Shalev U, Morales M, Hope B, Yap J, Shaham Y. Time-dependent changes in extinction behavior and stress-induced reinstatement of drug seeking following withdrawal from heroin in rats. Psychopharmacology. 2001;156:98-107.

20. Theberge FR, Pickens CL, Goldart E, Fanous S, Hope BT, Liu QR, et al. Association of time-dependent changes in mu opioid receptor $\mathrm{mRNA}$, but not BDNF, TrkB, or MeCP2 mRNA and protein expression in the rat nucleus accumbens with incubation of heroin craving. Psychopharmacology. 2012;224:559-71.

21. Bedi G, Preston KL, Epstein DH, Heishman SJ, Marrone GF, Shaham Y, et al. Incubation of cue-induced cigarette craving during abstinence in human smokers. Biol Psychiatry. 2011;69:708-11.

22. Li P, Wu P, Xin X, Fan YL, Wang GB, Wang F, et al. Incubation of alcohol craving during abstinence in patients with alcohol dependence. Addict Biol. 2015;20:513-22.
23. Wang G, Shi J, Chen N, Xu L, Li J, Li P, et al. Effects of length of abstinence on decision-making and craving in methamphetamine abusers. PLoS ONE. 2013;8: e68791.

24. Parvaz MA, Moeller SJ, Goldstein RZ. Incubation of cue-induced craving in adults addicted to cocaine measured by electroencephalography. JAMA Psychiatry. 2016;73:1127-34.

25. Venniro M, Caprioli D, Shaham Y. Animal models of drug relapse and craving: From drug priming-induced reinstatement to incubation of craving after voluntary abstinence. Prog Brain Res. 2016;224:25-52.

26. Epstein $\mathrm{DH}$, Preston $\mathrm{KL}$. The reinstatement model and relapse prevention: a clinical perspective. Psychopharmacology. 2003;168:31-41.

27. Olds J, Olds ME. Positive reinforcement produced by stimulating hypothalamus with iproniazid and other compounds. Science. 1958;127:1175-76.

28. Warden CJ. Animal motivation: experimental studies on the albino rat. New York: Columbia University Press; 1931.

29. Jenkins TN, Warner LH, Warden CJ. Standard apparatus for the study of animal motivation. J Comp Psychol. 1926;6:361-82.

30. Cooper A, Barnea-Ygael N, Levy D, Shaham Y, Zangen A. A conflict rat model of cue-induced relapse to cocaine seeking. Psychopharmacology. 2007;194:117-25.

31. Peck JA, Wercberger R, Kariyeva E, Ranaldi R. Cue-induced resumption of heroin and cocaine seeking in rats using a conflict model of abstinence and relapse. Psychopharmacology. 2013;228:651-58.

32. Saunders BT, Yager LM, Robinson TE. Cue-evoked cocaine "craving": role of dopamine in the accumbens core. J Neurosci. 2013:33:13989-4000.

33. Marchant NJ, Campbell EJ, Pelloux Y, Bossert JM, Shaham Y. Context-induced relapse after extinction versus punishment: similarities and differences. Psychopharmacology. 2019;236:439-48.

34. Krasnova IN, Marchant NJ, Ladenheim B, McCoy MT, Panlilio LV, Bossert JM, et al. Incubation of methamphetamine and palatable food craving after punishmentinduced abstinence. Neuropsychopharmacology. 2014;39:2008-16.

35. Khemiri L, Steensland P, Guterstam J, Beck O, Carlsson A, Franck J, et al. The effects of the monoamine stabilizer (-)-OSU6162 on craving in alcohol dependent individuals: A human laboratory study. Eur Neuropsychopharmacol. 2015;25:2240-51.

36. Natesan S, Svensson KA, Reckless GE, Nobrega JN, Barlow KB, Johansson AM, et al. The dopamine stabilizers (S)-(-)-(3-methanesulfonyl-phenyl)-1-propyl-piperidine [(-)-OSU6162] and 4-(3-methanesulfonylphenyl)-1-propyl-piperidine (ACR16) show high in vivo D2 receptor occupancy, antipsychotic-like efficacy, and low potential for motor side effects in the rat. J Pharmacol Exp Ther. 2006;318:810-8.

37. Rung JP, Rung E, Helgeson L, Johansson AM, Svensson K, Carlsson A, et al. Effects of (-)-OSU6162 and ACR16 on motor activity in rats, indicating a unique mechanism of dopaminergic stabilization. J Neural Transm (Vienna). 2008;115: 899-908.

38. Sonesson C, Lin CH, Hansson L, Waters N, Svensson K, Carlsson A, et al. Substituted (S)-phenylpiperidines and rigid congeners as preferential dopamine autoreceptor antagonists: synthesis and structure-activity relationships. J Med Chem. 1994:37:2735-53.

39. Fredriksson I, Wirf M, Steensland P. The monoamine stabilizer (-)-OSU6162 prevents the alcohol deprivation effect and improves motor impulsive behavior in rats. Addict Biol. 2019;24:471-84.

40. Steensland P, Fredriksson I, Holst S, Feltmann K, Franck J, Schilstrom B, et al. The monoamine stabilizer (-)-OSU6162 attenuates voluntary ethanol intake and ethanol-induced dopamine output in nucleus accumbens. Biol Psychiatry. 2012;72:823-31.

41. Caprioli D, Venniro M, Zeric T, Li X, Adhikary S, Madangopal R, et al. Effect of the novel positive allosteric modulator of metabotropic glutamate receptor 2 AZD8529 on incubation of methamphetamine craving after prolonged voluntary abstinence in a rat model. Biol Psychiatry. 2015:78:463-73.

42. Caprioli D, Venniro M, Zhang M, Bossert JM, Warren BL, Hope BT, et al. Role of dorsomedial striatum neuronal ensembles in incubation of methamphetamine craving after voluntary abstinence. J Neurosci. 2017;37:1014-27.

43. Venniro M, Zhang M, Caprioli D, Hoots JK, Golden SA, Heins C, et al. Volitional social interaction prevents drug addiction in rat models. Nat Neurosci. 2018; 21:1520-29.

44. Richardson NR, Roberts DC. Progressive ratio schedules in drug self-administration studies in rats: a method to evaluate reinforcing efficacy. J Neurosci Methods. 1996;66:1-11.

45. Venniro M, Zhang M, Shaham Y, Caprioli D. Incubation of methamphetamine but not heroin craving after voluntary abstinence in male and female rats. Neuropsychopharmacology. 2017;42:1126-35.

46. Airavaara M, Pickens $C L$, Stern AL, Wihbey KA, Harvey BK, Bossert JM, et al. Endogenous GDNF in ventral tegmental area and nucleus accumbens does not play a role in the incubation of heroin craving. Addiction. Biol. 2011;16:261-72.

47. Stewart J, Woodside B, Shaham Y. Ovarian hormones do not affect the initiation and maintenance of intravenous self-administration of heroin in the female rat. Psychobiology. 1996;24:154-59. 
48. Venniro M, Russell TI, Zhang M, Shaham Y. Operant social reward decreases incubation of heroin craving in male and female rats. Biol Psychiatry. 2019;86:848-56

49. Becker JB, Chartoff E. Sex differences in neural mechanisms mediating reward and addiction. Neuropsychopharmacology. 2019;44:166-83.

50. Shaham Y, Shalev U, Lu L, De Wit H, Stewart J. The reinstatement model of drug relapse: history, methodology and major findings. Psychopharmacology. 2003;168:3-20.

51. Epstein DH, Preston KL, Stewart J, Shaham Y. Toward a model of drug relapse: an assessment of the validity of the reinstatement procedure. Psychopharmacology. 2006;189:1-16

52. Katz JL, Higgins ST. The validity of the reinstatement model of craving and relapse to drug use. Psychopharmacology. 2003;168:21-30.

53. Marlatt GA. Do animal models provide a valid analogue for human drug lapse and relapse? Comment on Leri and Stewart (2002). Exp Clin Psychopharmacol. 2002;10:359-60

54. Vanderschuren LJM, Minnaard AM, Smeets JAS, Lesscher HMB. Punishment models of addictive behavior. Cur Opinion. Behav Sci. 2017;13:77-84.

55. Jean-Richard-Dit-Bressel P, Killcross S, McNally GP. Behavioral and neurobiological mechanisms of punishment: implications for psychiatric disorders. Neuropsychopharmacology. 2018;43:1639-50.

56. Economidou D, Pelloux Y, Robbins TW, Dalley JW, Everitt BJ. High impulsivity predicts relapse to cocaine-seeking after punishment-induced abstinence. Biol Psychiatry. 2009;65:851-56.

57. Marchant NJ, Khuc TN, Pickens CL, Bonci A, Shaham Y. Context-induced relapse to alcohol seeking after punishment in a rat model. Biol Psychiatry. 2013;73:256-62.

58. Panlilio L, Thorndike E, Schindler C. Reinstatement of punishment-suppressed opioid self-administration in rats: an alternative model of relapse to drug abuse. Psychopharmacology. 2003;168:229-35.

59. Farrell MR, Ruiz CM, Castillo E, Faget L, Khanbijian C, Liu SY, et al. Ventral pallidum is essential for cocaine relapse after voluntary abstinence in rats. Neuropsychopharmacology. 2019;44:2174-85.

60. Pelloux Y, Minier-Toribio A, Hoots JK, Bossert JM, Shaham Y. Opposite effects of basolateral amygdala inactivation on context-induced relapse to cocaine seeking after extinction versus punishment. J Neurosci. 2018;38:51-59.

61. de Wit H, Epstein DH, Preston KL. Does human language limit translatability of clinical and preclinical addiction research? Neuropsychopharmacology. 2018;43: 1985-88.

62. Azrin NH, Holz WC. Punishment. In: Honig WK, editor. Operant behavior: areas of research and application. Englewood Cliffs, N.J: Prentice-Hall; 1966. p. 380-447.

63. Piazza PV, Le Moal M. The role of stress in drug self-administration. Trends Pharmacol Sci. 1998;19:67-74.

64. Mantsch JR, Baker DA, Funk D, Le AD, Shaham Y. Stress-induced reinstatement of drug seeking: 20 years of progress. Neuropsychopharmacology. 2016;41:335-56.

65. Glynn RM, Rosenkranz JA, Wolf ME, Caccamise A, Shroff F, Smith AB, et al. Repeated restraint stress exposure during early withdrawal accelerates incubation of cue-induced cocaine craving. Addict Biol. 2018;23:80-89.

66. Badiani A. Substance-specific environmental influences on drug use and drug preference in animals and humans. Curr Opin Neurobiol. 2013;23:588-96.

67. Badiani A, Belin D, Epstein D, Calu D, Shaham Y. Opiate versus psychostimulant addiction: the differences do matter. Nat Rev Neurosci. 2011;12:685-700.

68. Montanari C, Stendardo E, De Luca MT, Meringolo M, Contu L, Badiani A. Differential vulnerability to relapse into heroin versus cocaine-seeking as a function of setting. Psychopharmacology (Berl). 2015;232:2415-24.

69. Carlsson ML, Carlsson A, Nilsson M. Schizophrenia: from dopamine to glutamate and back. Curr Med Chem. 2004;11:267-77.

70. Bossert JM, Marchant NJ, Calu DJ, Shaham Y. The reinstatement model of drug relapse: recent neurobiological findings, emerging research topics, and translational research. Psychopharmacology. 2013;229:453-76.

71. Renier N, Adams EL, Kirst C, Wu Z, Azevedo R, Kohl J, et al. Mapping of brain activity by automated volume analysis of immediate early genes. Cell. 2016;165:1789-802.

72. De Vries TJ, Shippenberg TS. Neural systems underlying opiate addiction. J Neurosci. 2002;22:3321-25.
73. De Vries TJ, Schoffelmeer AN, Binnekade R, Raaso H, Vanderschuren LJ. Relapse to cocaine- and heroin-seeking behavior mediated by dopamine D2 receptors is time-dependent and associated with behavioral sensitization. Neuropsychopharmacology. 2002;26:18-26.

74. Shaham Y, Stewart J. Effects of opioid and dopamine receptor antagonists on relapse induced by stress and re-exposure to heroin in rats. Psychopharmacology. 1996;125:385-91.

75. Galaj E, Manuszak M, Babic S, Ananthan S, Ranaldi R. The selective dopamine D3 receptor antagonist, SR 21502, reduces cue-induced reinstatement of heroin seeking and heroin conditioned place preference in rats. Drug Alcohol Depend. 2015;156:228-33.

76. Lai $M$, Chen W, Zhu $H$, Zhou X, Liu H, Zhang F, et al. Low dose risperidone attenuates cue-induced but not heroin-induced reinstatement of heroin seeking in an animal model of relapse. Int J Neuropsychopharmacol. 2013;16:1569-75

77. Yue K, Ma B, Chen L, Tian X, Ru Q, Gan Y, et al. L-Stepholidine, a naturally occurring dopamine D1 receptor agonist and D2 receptor antagonist, attenuates heroin self-administration and cue-induced reinstatement in rats. Neuroreport. 2014:25:7-11.

78. Bossert JM, Poles GC, Wihbey KA, Koya E, Shaham Y. Differential effects of blockade of dopamine D1-family receptors in nucleus accumbens core or shell on reinstatement of heroin seeking induced by contextual and discrete cues. Neurosci. 2007;27:12655-63.

79. Vander Weele CM, Porter-Stransky KA, Mabrouk OS, Lovic V, Singer BF, Kennedy $\mathrm{RT}$, et al. Rapid dopamine transmission within the nucleus accumbens: dramatic difference between morphine and oxycodone delivery. The European journal of neuroscience. 2014;40:3041-54.

80. Gottas A, Boix F, Oiestad EL, Vindenes V, Morland J. Role of 6-monoacetylmorphine in the acute release of striatal dopamine induced by intravenous heroin. Int J Neuropsychopharmacol. 2014;17:1357-65.

81. Robinson TE, Becker JB. Enduring changes in brain and behavior produced by chronic amphetamine administration: a review and evaluation of animal models of amphetamine psychosis. Brain Res. 1986;396:157-98.

82. Becker JB, Hu M. Sex differences in drug abuse. Frontiers Neuroendocrinol. 2008;29:36-47.

83. Roth $M E$, Cosgrove $\mathrm{KP}$, Carroll ME. Sex differences in the vulnerability to drug abuse: a review of preclinical studies. Neurosci Biobehav Rev. 2004;28:533-46.

84. Mello NK, Negus SS. Preclinical evaluation of pharmacotherapies for treatment of cocaine and opioid abuse using drug self-administration procedures. Neuropsychopharmacology. 1996;14:375-424.

85. Van Ree JM, Ramsey N. The dopamine hypothesis of opiate reward challenged. Eur J Pharmacol. 1987;134:239-43.

86. Van Ree JM, Gerrits MA, Vanderschuren LJ. Opioids, reward and addiction: An encounter of biology, psychology, and medicine. Pharmacological reviews. 1999;51:341-96.

87. Ettenberg A, Pettit HO, Bloom FE, Koob GF. Heroin and cocaine intravenous selfadministration in rats: mediation by separate neural systems. Psychopharmacology (Berl). 1982;78:204-09.

88. Stinus L, Cador M, Le Moal M. Interaction between endogenous opiods and dopamine within the nucleus accumbens. Ann N Y Acad Sci. 1992;654:254-73.

89. Kloberg A, Constantinescu R, Nilsson MK, Carlsson ML, Carlsson A, Wahlstrom J, et al. Tolerability and efficacy of the monoaminergic stabilizer (-)-OSU6162 (PNU96391A) in Huntington's disease: a double-blind cross-over study. Acta Neuropsychiatr. 2014;26:298-306.

90. Tedroff J, Ekesbo A, Sonesson C, Waters N, Carlsson A. Long-lasting improvement following (-)-OSU6162 in a patient with Huntington's disease. Neurology. 1999;53:1605-06.

91. Berginstrom N, Nordstrom P, Schuit R, Nordstrom A The effects of (-)-OSU6162 on chronic fatigue in patients with traumatic brain injury: a randomized controlled trial. J Head Trauma Rehabil. 2017;32:E46-e54.

92. Johansson B, Carlsson A, Carlsson ML, Karlsson M, Nilsson MK, Nordquist-Brandt $E$, et al. Placebo-controlled cross-over study of the monoaminergic stabiliser (-)-OSU6162 in mental fatigue following stroke or traumatic brain injury. Acta Neuropsychiatr. 2012;24:266-74. 